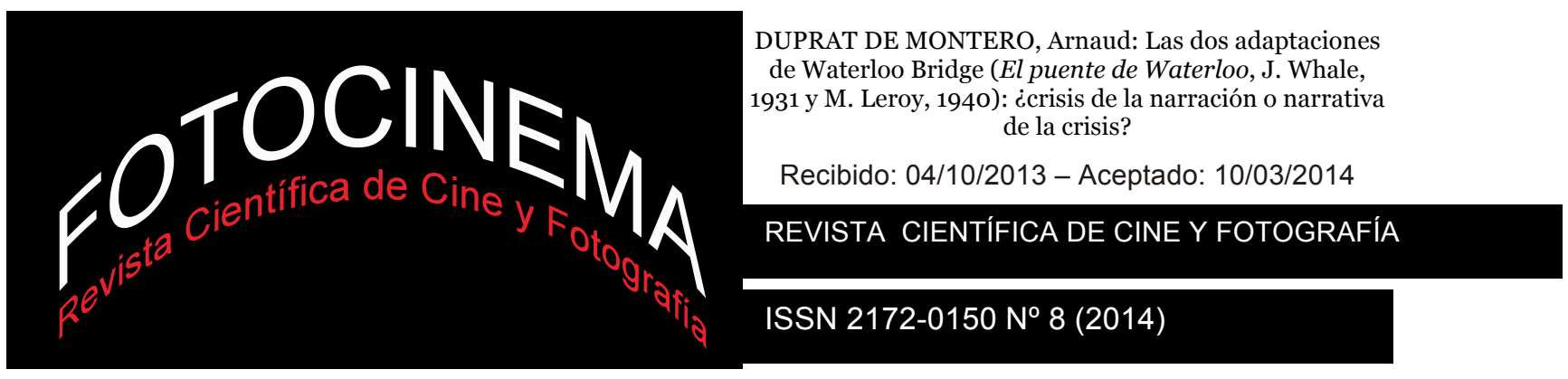

\title{
LAS DOS ADAPTACIONES DE WATERLOO BRIDGE (EL PUENTE DE WATERLOO, J. WHALE, 1931 Y M. LEROY, 1940): ¿CRISIS DE LA NARRACIÓN O NARRATIVA DE LA CRISIS?
}

\section{THE TWO ADAPTATIONS OF WATERLOO BRIDGE (WATERLOO BRIDGE, J. WHALE, 1931 AND M. LEROY, 1940): CRISIS OF THE NARRATION OR NARRATION OF THE CRISIS?}

\author{
Arnaud Duprat de Montero \\ Université Rennes 2 (Francia)
}

\section{Resumen:}

Se suelen conocer las dos adaptaciones de la obra de teatro Waterloo Bridge (Robert E. Sherwood, 1930) por parte de James Whale (1931) y de Mervyn LeRoy (1940) como un buen ejemplo de los cambios ideológicos y morales que sufrió Hollywood a partir de la aparición del código de censura Hays en 1934. Sin embargo, si la comparación de las dos películas pone de realce, en efecto, estos cambios que podemos asimilar a una crisis de la narración cinematográfica, al contar la historia de amor entre un soldado y una prostituta durante la gran crisis política que fue la Primera Guerra Mundial, estos filmes nos hablan sobre todo de su actualidad -la gran depresión en el caso de Whale y el principio de la Segunda Guerra Mundial con LeRoy- y pertenecen a la narrativa cinematográfica de la crisis. Más que el mero resultado de un compromiso político y social, estas películas son también auténticas obras de autores en simbiosis con su época. En este sentido, la versión de LeRoy marca el principio de una mezcla entre melodrama y cine negro que determinará este género a lo largo de las décadas de los cuarenta y de los cincuenta.

\begin{abstract}
:
According to many commentators the two adaptations of the play Waterloo Bridge (Robert E. Sherwood, 1930) directed by James Whale (1931) and Mervyn LeRoy (1940) represent a good example of ideological and moral changes undergone by Hollywood after the publication of the Hays Code in 1934. However, whilst a comparison of the two films does indeed throw light upon the changes in question, which sprung from a crisis in cinematographical narration, each work speaks particularly eloquently of its own epoch through the story of love between a prostitute and a soldier during the great political crisis of the First World War; the two films can thus be considered as cinematographical narrations of crisis. Far more than the simple result of political and social engagement, these films are also the works of directors in touch with their time. In this way, LeRoy's version marks the beginning of the meeting between melodrama and film noir which would define the latter throughout the 1940 and 1950 s.
\end{abstract}

\section{Palabras clave:}

Guerra; Hays; Hollywood; J. Whale; M. LeRoy; V. Leigh

\section{Keywords:}

Guerra; Hays; Hollywood; J. Whale; M. LeRoy; V. Leigh 


\section{Introducción}

En cuanto se estrenó, en 1930, la obra de teatro de Robert E. Sherwood, Waterloo Bridge, Hollywood quiso llevarla a la gran pantalla. Aunque no fue un gran éxito en Broadway, esta obra, al contar la historia de amor entre un joven soldado de clase alta y una prostituta en Londres durante la Primera Guerra Mundial, reunía bastantes elementos para constituir un buen melodrama popular ${ }^{1}$. Sin embargo, en la medida en que se estrenó en 1931 el 1 de septiembre-, es decir sólo tres años antes de la aparición del código de censura llamado código Hays -el 1 de julio de 1934-, la adaptación dirigida por James Whale, a pesar de funcionar bien en taquilla, no pudo volver a estrenarse y quedó en la historia como emblemática de las libertades del cine hollywoodiense pre-código. En este sentido, se suele considerar el remake de Mervyn LeRoy que se estrenó casi diez años más tarde -el 16 de mayo de 1940-, como un buen ejemplo de las consecuencias del código Hays, debido a los cambios importantes efectuados en la representación de la heroína y en el relato de su historia ${ }^{2}$. No obstante, sin intentar negar del todo el papel desempeñado por este código en la película de 1940, no debemos olvidarnos del contexto en el que Whale y LeRoy dirigieron sus películas. En efecto, si las dos sitúan la historia de amor de sus protagonistas durante la Primera Guerra Mundial, sus contextos de creación no fueron menos dramáticos: la primera se realizó en los primeros años de la crisis económica debida al crack de la bolsa neoyorquina el 24 de octubre de 1929, y la segunda se rodó de

1. Así Jean-Loup Bourget define el melodrama hollywoodiense: «On définira comme "mélodrame" tout film hollywoodien qui présente les caractéristiques suivantes : un personnage de victime (souvent une femme, un enfant, un infirme), une intrigue faisant appel à des péripéties providentielles ou catastrophiques, et non au seul jeu des circonstances réalistes ; enfin, un traitement qui met l'accent soit sur le pathétique et la sentimentalité (faisant partager au spectateur, au moins en apparence, le point de vue de la victime), soit sur la violence des péripéties, soit (le plus souvent) tour à tour sur ces deux éléments, avec les ruptures de ton que cela implique.» (Bourget, 1994, pp. 12-13).

2. Hasta recientemente, es con este enfoque que se suele hablar de estas dos películas. En 1998, Jean-Pierre Mattei consideraba que «l'esprit puritain de LeRoy dénature le vrai sujet de la pièce mais donne plus de force à ce superbe mélodrame» (Mattéi, 1998, p. 174). Cuando se estrenó en Francia y en 2012, el dvd con los dos films juntos, Alain Masson escribió que «le second film présente d'ailleurs une relation pertinente et curieuse avec le premier qu'il enveloppe dans son flash-back en corrigeant ses indécences abruptes» (Masson, 2012, p. 78) e Yves Alion añadió que «au début des années 30 (...) la déchéance de Myra pouvait (...) être décrite (...), ce qu'il n'était plus possible de faire dans les années 40» (Alion, 2012, pp. 132-133). 
diciembre de 1939 a enero de 1940, o sea sólo unas semanas después de la declaración de guerra entre Reino Unido y Alemania. Entonces, cabe interrogarse sobre el papel desempeñado por estos contextos -una crisis económica con Whale, una crisis política con LeRoy- en los dos films, tanto en su representación de la Primera Guerra Mundial -otra crisis políticacomo en su propio relato. Dicho de otro modo, estas películas no serían solamente ejemplos de una crisis de la narración por culpa de un código de censura, sino también ejemplos de narrativa de crisis ${ }^{3}$. Después de haber estudiado los cambios en la película de LeRoy, con relación a la de Whale, que respetan el código Hays, veremos cómo los dos films representan la Primera Guerra Mundial, tanto en los diálogos o situaciones, como en su puesta en escena de una ciudad atacada. Estas comparaciones serán necesarias a la hora de establecer un balance de la importancia del crack de 1929 y de la Segunda Guerra Mundial en su creación, sin olvidarnos de considerar las dos películas dentro de las filmografías de sus directores James Whale y Mervyn LeRoy, éste siendo el iniciador del cine negro, y también, en el caso de esta adaptación, de su actriz protagonista, la británica Vivien Leigh.

\section{Marco teórico y metodología}

El estreno reciente en DVD de la película Waterloo Bridge (Mervyn LeRoy, 1940) -en España (2009) como en EEUU (2009) y sobretodo en Francia (2012) ya que viene en esta edició con la otra versión de James Whale-, ha permitido descubrir de nuevo estos dos filmes hasta entonces poco visibles. El DVD francés se vio acompañado por la publicación de dos artículos -de Alion y de Masson- que, al comparar las películas de LeRoy y de Whale, se sitúan en la continuidad de un artículo de Mattéi de 1998 y el análisis que propone McLean de la versión de 1940 en su libro Dying Swans and Madmen en 2008. Si las referencias son todavía limitadas sobre estos dos

3. Por estas razones, no hablaremos de la tercera adaptación de la obra de Sherwood, titulada Gaby (Curtis Bernhardt, 1956), estrenada en un periodo de auge económico en la que el código Hays, en una nueva versión más blanda, permitía representar la prostitución con más libertad. 
filmes, el enfoque elegido consiste siempre en considerarlos como buenos ejemplos de las consecuencias de la aparición del código Hays en 1934. Mi objetivo con este trabajo es -en la continuidad de la entrevista de RenéOlivier Veillon que ya va más allá en los extras del DVD francésinterrogarme sobre la pertinencia de este enfoque e intentar ver si la influencia del contexto económico, social y político no permite también proponer otras lecturas fructíferas que abran las posibilidades interpretativas. Además, las características de cada película interpretadas como consecuencias del código Hays podrían ser también rasgos artísticos de la evolución de sus autores. Con este objetivo, mi trabajo presenta un enfoque no solamente histórico (con referencias al artículo de Mainer en Fotocinema) sino también textual - recurriendo a referencias sobre géneros (Bourget, Silver \& Ward) o sobre la estructura del relato fílmico (Mouren), y se basa en las pocas monografías dedicadas a Whale (Curtis), LeRoy (su propia autobiografía) y - bajo la influencia de los estudios actorales que consideran el actor como un autor posible - Vivien Leigh (Dent, Walker).

\section{La prostitución: de una profesión cualquiera a la decadencia moral}

Como lo hemos dicho, se conoce Waterloo Bridge de James Whale como un ejemplo del cine hollywoodiense pre-código Hays. La película debe esta fama, sin duda alguna, al hecho de tener como protagonista a Myra -Mae Clarke-, una prostituta londinense. Si el cine de Hollywood siguió filmando personajes de prostitutas en sus films después de 1934, se nota su época en Waterloo Bridge en su representación de la prostitución. En efecto, Myra se prostituye sin experimentar un verdadero sentimiento de decadencia moral o social. Para ella, en un contexto laboral difícil debido a la guerra, la prostitución es solamente una etapa profesional y no cabe duda para ella que, en cuanto termine el conflicto, volverá a su antiguo trabajo, el de bailarina de cabaré. De hecho, aparentemente, estas dos profesiones no son tan diferentes. Al principio de la película, cuando Myra es aún bailarina, la vemos al final de una función, ofreciendo su cuerpo poco vestido a las miradas del 
público y después, en los camerinos, mientras se está cambiando con sus compañeras y que están todas en ropa interior ${ }^{4}$, la heroína admite claramente ser también, en sus relaciones sentimentales, una mujer mantenida. Más tarde, cuando conoce a Roy -Kent Douglass-, enseguida lo invita a cenar en su casa y no le molesta charlar con él tumbado a su lado sobre la cama5. Luego, la película filma explícitamente a Myra con sus clientes en Trafalgar Square o a otras prostitutas besándose con hombres, en la oscuridad nocturna de los parques -planos desde luego imposibles después de 1934-, como efectuando un trabajo cualquiera. En este sentido, es importante notar que en esta versión, la madre de Roy -Enid Bennettestá en contra del matrimonio de la pareja desde el principio, por pensar solamente que los novios son distintos socialmente, al ser Myra solamente bailarina. Al enterarse de la verdad ${ }^{6}$, casi no la vemos más ofendida, como si al final, bailarina y prostituta fuesen casi el mismo oficio. Por eso Myra no considera el matrimonio con Roy como una redención posible, sino solamente como una posibilidad de abandonar la prostitución y, si le cuesta confesar la verdad al joven, es porque no quiere reconocer la importancia de sus apuros económicos7.

A pesar de que, por supuesto, estos apuros sigan presentes en la versión de Mervyn LeRoy, lo que predomina en las vacilaciones de su heroína ante la petición de mano de Roy, es el sentimiento de decadencia moral. En 1940, Myra -Vivien Leigh- no podía ser, según el código Hays, una prostituta asumida $^{8}$ ya que se admitía este tipo de personajes solamente con un

\footnotetext{
4. Notemos que se ve mucho la ropa interior femenina en este film: cuando Myra habla de Roy con su amiga y vecina, la prostituta Kitty -Doris Lloyd-, ésta está lavando a mano bragas, medias y sujetadores. Luego, cuando Myra vuelve a casa por la mañana después de prostituirse, la vemos quitarse las medias.

5. Esta posición se repetirá después en un diálogo entre Roy y Kitty.

6. Notemos que esta escena, en comparación con la película de LeRoy, se beneficia aquí de un diálogo más explícito. Al explicar cómo conoció a Roy, Myra dice: «I picked Roy up on Waterloo Bridge.». En el remake, la futura suegra -Lucile Watson- entiende la verdad cuando la heroína le dice que es muy "naive" si piensa que su problema es sólo otro hombre. 7. Bien lo vemos cuando rechaza cualquier regalo o ayuda económica por parte de Roy, ya que le recuerda enseguida la diferencia social entre los dos: si la familia de él es acomodada, Myra es hija de «drunkards».

8. El código Hays presenta dos listas de temas: los «don'ts» -es decir los temas tajantemente prohibidos- y los «Be Carefuls»-temas con los que hay que llevar mucho cuidado-. La prostitución -«16. The sale of women, or of a woman selling her virtue»- forma parte de éstos.
} 
enfoque moral que subrayara su decadencia. Por eso, cuando empieza la historia, Myra es una joven primera bailarina que forma parte, con su amiga Kitty -Virginia Field-, de un ballet que representa El lago de los cisnes de Tchaïkovski9. Además de esta profesión mucho más honrada, diversos personajes subrayan en varios momentos su juventud, su bondad y, sobre todo, su inocencia. De hecho, el propio día del encuentro con Roy -Robert Taylor-, cuando éste acude al teatro para invitar a Myra a cenar fuera, Kitty le advierte que es «a sweet child». Aunque los padres de Myra fuesen profesores humildes de Birmingham ${ }^{10}$, tanto este aspecto como su profesión no impiden a la joven casarse con Roy y, sin la marcha anticipada del regimiento de éste para Francia, la pareja hubiera contratado matrimonio y la heroína hubiera esperado el final de la guerra con su suegra en Escocia. Durante la ausencia de Roy, se acumulan los acontecimientos dramáticos que la llevan a la prostitución: el despido del ballet después de haber faltado una función por despedirse de Roy, el paro y, sobre todo, la publicación errónea del nombre de éste en la lista de los soldados fallecidos que provoca la desesperación de una heroína ya hambrienta. Se reúne la pareja en Waterloo Station cuando él vuelve del frente y ella está buscando clientes entre los soldados recién llegados. Con Mervyn LeRoy, un dilema moral sustituye el aspecto social en la segunda parte -aunque unas amigas celosas de Roy consideran a Myra de manera un poco despectiva por haber sido bailarina- y la heroína no elige enseguida entre contarlo todo a Roy y librarse, o callarse y encontrar la redención al hacerle feliz. Sin embargo, al final, Myra - «too honest», como lo dice Kitty- no puede con la mentira y decide huir de Escocia después de haber confesado la verdad a la que iba a ser su suegra Lucile Watson-. Al día siguiente, se suicida en el puente de Waterloo, tirándose bajo una ambulancia, impidiendo así para siempre la felicidad de

9. Por supuesto, la elección de este ballet es significativa. Ya que en esta obra Tchaïkovski cuenta, como lo puntaliza Olivier-René Veillon «le sacrifice même du corps», esta referencia intertextual anticipa el sacrificio de su propio cuerpo que realizará Myra prostituyéndose. Citado en los extras del dvd (LeRoy, 2012). Adrienne McLean añade: «(...) the performance (...) is only included, to provide a way for Roy to recognize Myra as a beautiful, otherworldly creature with wings and to fall in love with her. He will be unable to possess her ever, and this too is supported by the versions of Swan Lake then being performed theatrically around the United States.» (McLean, 2008, p. 82).

10. Notemos que la Myra de LeRoy lleva un apellido -Lester- cuando la de Whale da solamente su seudónimo de artista: Deauville. 
Roy. De esta forma, en el remake de 1940, los dos protagonistas son ante todo víctimas de guerra, ya que ésta los separa y hunde a la heroína con la supuesta muerte de su novio. El aspecto económico y material de la situación de Myra, sin desaparecer del todo, se vuelve secundario para dejar más importancia al tema del hambre -mientras que, con Whale, la principal preocupación de Myra es el alquiler- y la desesperación. La prostitución ya no es una actividad laboral como otra, sino un primer paso hacia el suicidio. De hecho, Myra conoce a su primer cliente en el puente de Waterloo cuando está mirando el agua, como pensando ya en poner fin a su vida. En este aspecto, es fundamental puntualizar que si la Myra de Whale muere también al final del film en el puente, es como víctima de un bombardeo y después de haber hablado con Roy quien le perdona su pasado. Aunque podamos ver este final como el reflejo de una era pre-código Hays ${ }^{11}$, el hecho de obtener el perdón de Roy y de seguir con el proyecto de la boda, representa a Myra como una joven que lucha por su futuro y que logra superar sus problemas, es decir una imagen radicalmente opuesta a la heroína de LeRoy.

\section{De la Primera a la Segunda Guerra Mundial}

$\mathrm{Si}$, en 1940, la representación de Myra como víctima de guerra respeta el código, no nos olvidemos de que esta película se rodó, como lo hemos dicho, al principio de la Segunda Guerra Mundial. Una diferencia importante en la

\footnotetext{
11. En efecto, si el código Hays es de 1934, ya en 1922 se había creado la Motion Pictures Producers and Distributors Association, presidida por el propio William Hays. No podía prohibir todavía, sino que emitía «recomendaciones», lo que favoreció, por supuesto, la auto-censura. Whale comenta así su final: «There could never be a happy ending. The tragedy is not in the life of the woman has led, but the fact she falls in love» (Curtis, 2003, p. 123). El final de Whale -y este análisis-, similar a la obra de Sherwood, refleja este contexto moral en el que ya, aparentemente, un final feliz era imposible para una prostituta: notemos que, de la heroína muerta, aparece en la pantalla solamente su prenda de piel, que vemos a menudo a lo largo de la película y que remite, metonímicamente, a sus actividades de prostituta. En el remake de LeRoy, vemos el mismo tipo de piel blanca en la habitación de Kitty. Cuando muere Myra al final, en vez de una prenda, la cámara destaca el talismán que había regalado a Roy, lo que desencadenó su destino trágico. Si un símbolo de prostitución concluye el film de Whale, es un símbolo del sacrificio de la heroína que cierra la película de LeRoy. De hecho, cuando la película estaba a punto de estrenarse, Hays la vio -el 24 de agosto- e hizo « a warning that a film about prostitution would have a rough time getting past the numerous state and local censor boards that dotted the country. Chicago, as it turned out, made the most deletions (...) New York and Pennsylvania made similar cuts (...) Reluctantly, Whale eliminated the scene of Myra in the artist's studio on Hays' assurances it would be cut in most cities» (Curtis, 2003, p. 125).
} 
película de LeRoy, respecto a Sherwood y Whale, es que el relato de la película empieza el 3 de septiembre de 1939 cuando Reino Unido entra en guerra contra Alemania. Roy, un coronel, al pasar por el puente de Waterloo, recuerda a Myra y un largo flash-back nos cuenta la historia de los dos durante la Primera Guerra Mundial. Esta estructura narrativa permite una comparación explícita entre las dos guerras y, a lo largo de la película, podemos olvidarnos de que esta historia pasa en 1914-1918 ya que la reconstitución no subraya mucho el cambio temporal -salvo algunos coches que vemos en unas pocas escenas-. Incluso la manera de vestir y los peinados son muy similares a los que estaban de moda en 1940. Para el espectador coetáneo, la película parece ser muy actual sobre todo que nos enteramos muy poco de los distintos acontecimientos o de la evolución de la contienda: nunca se nombran batallas, fechas, éxitos o fracasos de los países involucrados... Cuando, por fin, el flash-back acaba y volvemos a 1939, el desfase temporal no anula la empatía creada por el destino trágico de Myra y nos permite entender que este tipo de historia está a punto de volver a producirse. Otra diferencia importante entre el remake de 1940 y las versiones de Sherwood y Whale es que los dos protagonistas ya no son norteamericanos, sino británicos. Recordemos que, en 1940, todavía Estados Unidos no participaban en la guerra. Si los Myra y Roy de 1940 hubieran sido norteamericanos, el impacto sobre el espectador hubiera sido distinto ya que, al ser Estados Unidos un país neutral, su historia no podía repetirse en 1940 y entonces la historia no hubiera sido percibida con la misma actualidad. Esta empatía provocada asimila la película al intento colectivo, por parte de varios cineastas dentro y fuera de Hollywood, de llamar la atención de los estadounidenses sobre lo que estaba pasando en Europa.

El contexto de producción siendo totalmente distinto en 1931, James Whale pudo respetar la obra original de Sherwood y conservar la nacionalidad estadounidense de sus dos protagonistas. Solamente trece años después del final de la Primera Guerra Mundial, el personaje de Roy recuerda al público norteamericano que los Estados Unidos lucharon y, gracias a Myra, que sufrieron con el resto del mundo. Sin darnos tampoco informaciones concretas que permitan situar exactamente los acontecimientos de la 
historia, la guerra está más presente en la pantalla con Whale que con LeRoy: vemos los aviones, los bombardeos, la muchedumbre aterrorizada... Del mismo modo, la guerra, Alemania, el «Kaiser» y sus pérdidas humanas son el tema de varias conversaciones como por ejemplo en casa de Roy con su familia. A pesar de esta implicación subrayada, los dos héroes, como lo hemos dicho, no actúan nunca como víctimas, sino que salen adelante y no pierden la esperanza de un futuro mejor. Si esta representación respeta el recuerdo muy patriótico de la Primera Guerra Mundial -ya que, como todo el mundo lo sabía, Estados Unidos salieron victoriosos del conflicto-, podría también depender de su contexto de producción y desempeñar un papel importante para el estado de ánimo del público de 1931.

\section{De la esperanza a un melodrama propagandístico de autores}

Cuando se estrena Waterloo Bridge de James Whale, el público norteamericano lleva ya casi dos años padeciendo la crisis económica y, antes de la investidura de Roosevelt el 4 de marzo de 1933 y la esperanza que provocará su «New Deal», el futuro es todavía muy pesimista. Aunque el cine fuese una de las pocas industrias que la crisis no perjudicó realmente, como lo puntualizó Carmen Mainer, «(...) las fuentes coinciden en destacar el tono más bien trágico de los films rodados en los primeros años de la depresión» (Mainer, 04/2013, p. 178). Waterloo Bridge refleja este contexto ${ }^{12}$. Los problemas financieros son el tema principal de la mayoría de los diálogos: Myra recibe varias veces la visita de la dueña de su casa -Ethel Griffies- para pedirle el alquiler ${ }^{13}$, le cortan la luz... Lo hemos dicho, la honra de la joven no le permite reconocer su falta de dinero ni aceptar la ayuda financiera de Roy. Por eso, rechaza el dinero de éste, aunque tenga que volver a salir después a prostituirse. Esta presencia de las preocupaciones cotidianas de 1931 tiene

\footnotetext{
12. Un contexto que tuvo consecuencias en el propio rodaje: «The studio had just come off a six-week production shutdown, and Whale was under pressure to bring the film in at an unrealistically low cost. Universal, which had reported the biggest loss of its history for the fiscal year ended november I, 1930, was broke (...) Rumors were that Bank of America, a principal source of funding for the company, was ready to fold.» (Curtis, 2003, p. 121).

13. Notemos que, en le película de 1940, Ethel Griffies desempeña de nuevo este mismo papel. Sin embargo, como estos aspectos puramente materialistas son menos importantes, la actriz tiene solamente un cameo de unos segundos.
} 
como consecuencia el hecho de que el espectador se olvide a veces de que esta historia pasa en los años 10 y que la vea como actual. Como en la película de LeRoy, la puesta en escena no pone de realce los elementos que hubieran podido indicar el desfase temporal y el vestuario no es realmente distinto de la moda de 1931. De la misma manera, cuando empieza la película, el relato no indica la fecha y los acontecimientos parecen respetar la evolución de la crisis económica. Así, al principio, el ambiente es alegre, el teatro en el que trabaja Myra está lleno y su amante del momento, según lo que cuenta, le hace regalos costosos. La secuencia siguiente pasa dos años después y la heroína ya se prostituye. Gracias a este cambio de situación, el espectador imagina lógicamente que esta nueva secuencia representa la actualidad -es decir, 1931- y la anterior 1929, justo antes del crack. Es solamente cuando se ve a Myra en el puente de Waterloo, bajo los bombardeos, que el espectador entiende que esta historia pasa durante la Primera Guerra Mundial. En la medida en que la actualidad no desaparece del todo a pesar del carácter histórico de la historia, estos personajes no pueden ser pesimistas o derrotistas como la Myra de 1940, ya que no debían hundir del todo el estado de ánimo del espectador de 1931. Así, demuestran que, a pesar de las dificultades, hay que seguir luchando. Por eso, Myra no se suicida, sino que muere como víctima de guerra en un momento en que, lo hemos dicho, acaba de alcanzar su objetivo con Roy ${ }^{14}$. Hasta el bombardeo, existe la esperanza de un final feliz para los dos protagonistas y este aspecto determina la estructura del relato: en estas circunstancias, era imposible empezar con el final y contar la historia gracias a un flash-back.

La estructura del relato es, desde luego, la diferencia esencial entre las dos películas ya que determina el tono de cada una. El flash-back de Mervyn LeRoy, si establece un paralelismo explícito entre las dos guerras mundiales con efectos propagandísticos, convierte también lo que era un mero melodrama en una verdadera tragedia. Como lo subraya Yannick Mouren, «le tragique n'est pas loin du mélodrame. La compatibilité entre le flash-back

14. Puntualicemos que, en el final de Sherwood, Myra no muere. Sin embargo, da pruebas de una actitud suicida, al quedarse en el puente y al encender un cigarrillo para llamar la atención de los aviones alemanes (Leer: Sherwood, 1930, p. 173). 
(...) et le mélodrame n'est plus à démontrer. (...) Le propre des films "qui commencent par la fin" est qu'ils obligent le spectateur à ne plus se poser la question infantile par excellence "et après ?", mais celle plus adulte du "comment?" (...) Or, la tragédie pure, (...) celle de l'antiquité grecque, ne fonctionne pas du tout sur le suspens» (Mouren, 2005, p. 81). De esta forma, todo el flash-back de la película de LeRoy contesta a esta pregunta: ¿Cómo estos dos personajes van a conocer un final trágico mientras que su encuentro y los primeros días de su relación parecían ser la promesa de un final feliz ${ }^{15}$ ? Este fenómeno acentúa, por supuesto, el dramatismo y la empatía del espectador por los protagonistas ${ }^{16}$. Además, el carácter trágico de Waterloo Bridge de Mervyn LeRoy depende de la presencia de la muerte a lo largo de la historia. Como dijo Olivier-René Veillon, gracias al flash-back, «les personnages sont les fantômes d'eux-mêmes» (LeRoy, 2012), lo que puede explicar la falta de carnalidad en el remake de 1940, respecto al film de James Whale, más allá de una mera consecuencia del código Hays. Para Veillon, el propio puente de Waterloo cobra una dimensión metafórica, la de un puente «entre deux rives, deux époques, et le balcon qui surplombe le flux du temps qu'on ne peut retenir comme l'eau» (LeRoy, 2012). En este sentido, cabe notar que, en efecto, cada vez que Myra o Roy están pensando en el otro muerto, se encuentran en el puente, asomándose, con el reflejo del agua en sus rostros. Por fin, la muerte está presente en la puesta en escena: si Myra interviene por primera vez gracias a su voz en off cuando Roy la recuerda en 1939, otras voces acusmáticas -es decir, en este caso, fuera de campopresentan una dimensión mórbida, como la del primer cliente de Myra, que no veremos nunca en la pantalla, o la de los altavoces de la primera secuencia que anuncian el principio de la guerra y que parecen condenar a la muerte a todos los oyentes. De hecho, cuando Myra vuelve a ver a Roy en la estación de Waterloo, su asombro -filmado en primer plano- no da lugar

15. Aquí, el talismán desempeña un papel muy importante. Si, lo hemos dicho, es simbólico del sacrificio de Myra, sus apariciones a lo largo del flash-back -cuando Myra lo regala a Roy el primer día o cuando éste se lo devuelve en Escocia-, recuerdan al espectador el relato primero y que lo que estamos viendo es un flash-back que tendrá, como final, la soledad de Roy en 1939.

16. «Le spectateur (...) est fasciné par cette construction narrative en ce qu'elle suscite de la pitié envers les personnages» (Mouren, 2005, p. 82). 
inmediatamente a un contracampo, sino que el personaje masculino se queda varios segundos fuera de campo, lo que recuerda que, para Myra, sigue perteneciendo al mundo de los muertos. Por fin, la muerte está presente en la visión que ofrece la película de Londres, filmada en la mayoría de las escenas de noche, con niebla y muchas zonas de oscuridad ${ }^{17}$. Se nota menos la muchedumbre y el bullicio urbano con LeRoy que con Whale: salvo el primer plano de los londinenses que escuchan los altavoces, cuando luego se refugia la pareja protagonista en un abrigo por culpa de los bombardeos, la cámara se centra esencialmente sobre ellos. En el teatro en el que actúa Myra, el público, en su conjunto, queda en la oscuridad, y en el restaurante en el que los dos personajes bailan, una penumbra progresiva, según Veillon, «traduit cette menace (de la perte) : tout est évanescent, tout peut disparaître» (LeRoy, 2012).

Si la luz contrastada e inquietante del director de fotografía Joseph Ruttenberg ${ }^{18}$ participa en el tono trágico de la película, es también propia del trabajo de Mervyn LeRoy. En efecto, cabe recordar que este director, aunque empezó en 1928 rodando comedias, inició el cine negro en 1931 con su película Little Caesar. Además, gracias a su estética y su tono, Waterloo Bridge no fue sólo el caso particular de una influencia del cine negro en un melodrama, sino que fue el punto de partida de un fenómeno más amplio en el que estos dos géneros, al tener muchos puntos comunes, se mezclaron muchas veces al servicio de la propaganda pro-aliados, como lo explica JeanLoup Bourget: «Mais une évolution ( $\mathrm{du}$ mélodrame) beaucoup plus fondamentale se dessine pendant les années quarante, sous l'influence de facteurs propres combinée de facteurs extérieurs et de facteurs propres à l'histoire du cinéma, et affecte non seulement le mélodrame, mais l'ensemble de la production hollywoodienne et du système des genres : il s'agit de l'apparition du "film noir" ou thriller, qu'on peut définir succintement

\footnotetext{
17. Mervyn LeRoy recuerda el rodaje de la película: «(...) everything, or nearly everything, was supposedly set in the fog (...) we could suggest a locale, and the fog would be so thick we didn't have to be too specific with our sets. There was one scene of Vivien walking on a bridge. All we did was build part of the sidewalk and string some lights accross it, then fill the set with fog, and we had our bridge» (LeRoy, 1974, pp. 146-147).

18. Joseph Ruttenberg -que el propio LeRoy presenta como «one of the best» (LeRoy, 1974, p. 150)- había trabajado ya en varias películas de cine negro, entre ellas Fury (Fritz Lang, 1936).
} 
comme une série de films à l'intrigue criminelle, à l'atmosphère fataliste, aux décors souvent oppressants et aux éclairages vivement contrastés, voire néoexpressionnistes (...) Facteurs extérieurs : dès avant l'entrée des États-Unis dans le conflit, la guerre mobilise les énergies contre le nazisme et suscite la production d'ouvrages de propagande, qui constituent un des composants fondamentaux du film noir.» (Bourget, 1994, p. 258) A este fenómeno se puede también atribuir la estructura del flash-black que iba a convertirse en un rasgo característico del cine negro de los años cuarenta y cincuenta ${ }^{19}$. Waterloo Bridge sería, por todas estas razones, emblemática de su época y, al mismo tiempo, determinada por sus creadores: Mervyn LeRoy, como iniciador del cine negro, y también por su actriz protagonista, Vivien Leigh.

Ante todo, la dimensión propagandística de la película depende mucho de la presencia de esta actriz inglesa en la pantalla. Recordemos que, en 1940, llevaba todavía poco tiempo en Hollywood: llegó de Londres para reunirse con Laurence Olivier -su futuro esposo que estaba rodando Wuthering Heights de William Wyler- en diciembre de 1938. El día de Navidad de este mismo año, se enteró de que David O. Selznick la contrataba para Gone With the Wind (George Cukor, Victor Fleming, San Wood, 1939). Cuando Reino Unido entró en guerra, la pareja quiso volver enseguida a Londres, pero el gobierno inglés dijo a los directores y actores británicos que trabajaban en Estados Unidos que serían más útiles en Hollywood, rodando películas que sensibilizaran el público norteamericano a la causa del bando aliado. En este contexto, la actriz aceptó Waterloo Bridge ${ }^{2 O}$ y la producción británica de la London Films Lady Hamilton, dirigida por Alexander Korda (1941) con el visto bueno del propio Winston Churchill. El 27 de diciembre de 1940 -es decir antes del estreno de Lady Hamilton y a pesar de las recomendacionesVivien Leigh regresó con Laurence Olivier a Londres. Si la presencia de una

19. Yannick Mouren afirma que «le flash-back est la figure narrative de prédilection du film noir (...) Sur soixante-dix longs-métrages répertoriés par Alain Silver réalisés dans la grande période du film noir, entre 1941 et 1957 (...), j'ai dénombré vingt-cinq films non linéaires, plus du tiers (...) Le film en commençant par la fin confère au récit sa dimension tragique et exprime l'emprisonnement des personnages par le destin, deux caractéristiques du film noir» (Mouren, 2005, p. 130). Yannick Mouren cita a (Silver, 1987).

${ }^{20}$. «Waterloo Bridge (...) afforded Vivien at least proxy participation in the European crisis from which she and Olivier felt themselves so shamefully detached» (Walker, 1994, pp. 192193). 
verdadera inglesa en el reparto de Waterloo Bridge -Robert Taylor era norteamericano- da aún más realismo al drama vivido en tiempo de guerra por los británicos, la empatía creada se beneficia también del recuerdo de Gone With The Wind en la que Vivien Leigh encarnaba a Scarlett O'Hara, una heroína norteamericana durante la guerra de secesión cuyo recuerdo era todavía muy doloroso. De hecho, situaciones se repiten entre los dos films: el miedo al hambre, el venderse por dinero -sin llegar a prostituirse, recordemos que Scarlett se casa con su segundo esposo únicamente para poder conservar su casa-, el luto después de la muerte de un soldado... Si Scarlett sale victoriosa de la guerra, no es el caso de Myra, y esta comparación pone de realce la decadencia de ésta. Este cambio en el destino de las heroínas, significativo en una perspectiva propagandística, constituye también un momento importante en la filmografía de Vivien Leigh. Hasta entonces, la actriz británica había encarnado en Reino Unido heroínas que, como Scarlett, alcanzaban sus objetivos: citemos por ejemplo Liberty (St Martin's Lane, Tim Whelan, 1938) que empieza como ladrona y acaba estrella de musicales en los teatros de Londres -una situación similar a la de Myra al principio de Waterloo Bridge-, o Wanda (Twenty-One Days, Basil Dean, 1937) que tiene solamente veintiún días de felicidad con su novio Larry -Laurence Olivier- antes del encarcelamiento de éste y antes de que un acontecimiento inesperado demostrara su inocencia -otra situación que se parece a la de Myra a quien le queda sólo dos días antes de la salida de Roy para Francia ${ }^{21}$. Después de Waterloo Bridge, otra heroína de Vivien Leigh es víctima de la guerra: Emma Hamilton, en la película de propaganda de Korda -que recupera la estructura del flash-back-, se hunde socialmente a partir de la muerte de Lord Nelson -Laurence Olivier- hasta convertirse en vagabunda. Otras pierden una inocencia aparente - no nos olvidemos de que Emma, a pesar de su candor al principio, es prostituta- para alcanzar incluso una cierta perversidad -pensemos en Cleopatra (Caesar y Cleopatra, Gabriel Pascal, 1945) que acaba por matar a un hombre-. La decadencia moral, social y la desesperación absoluta son también el destino de otras heroínas

21. En otras películas británicas de guerra -Fire Over England, William K. Howard, 1937, Dark Journey, Victor Saville, 1937- sale también victoriosa. 
como Anna -Anna Karenina, Julien Duvivier, 1948- que se suicida de la misma manera que $\mathrm{Myra}^{22}$, Blanche Dubois $-A$ Streetcar Named Desire, Elia Kazan, 1951- que, después del suicidio de su esposo, se vuelve alcohólica y acumula los amantes -una situación que los otros personajes asimilan a la prostitución- o Karen Stone -The Roman Spring of Mrs Stone, José Quintero, 1961- que, al quedarse viuda, contrata los servicios de un joven prostituto -Warren Beatty- en Roma. Esta evolución de la persona de la actriz - con Scarlett y Blanche como las dos caras opuestas de una misma moneda- que otorga una coherencia autoral absoluta a una filmografía por otra parte escasa ${ }^{23}$, se produce al final de los años treinta y principio de los cuarenta -de GWTW a Lady Hamilton- con Waterloo Bridge como eslabón necesario.

\section{Conclusión}

La alta calidad artística de las dos primeras adaptaciones de Robert E. Sherwood por parte de James Whale y de Mervyn LeRoy, determinada por varios factores cinematográficos e históricos, demuestra el carácter muy reductor del enfoque adoptado casi siempre a la hora de hablar de ellas como de un díptico emblemático de las consecuencias del código Hays. Por supuesto, en la medida en que la película de Whale fue prohibida a partir de 1934 y que la de LeRoy respeta el código, lo son, pero su importancia en la historia del cine norteamericano va más allá. Por el hecho de cambiar la nacionalidad de los dos protagonistas y, gracias a un flash-back, de establecer un paralelismo entre las dos Guerras Mundiales, se puede añadir la película de LeRoy a la lista de los films de propaganda anti-fascista. Este aspecto crea un vínculo con el film de Whale que, al presentar a una heroína prostituta que sigue luchando y que encuentra el amor al final, no es solamente un ejemplo de cine sin censura, sino también una película que pone en escena el pasado para hablar de la actualidad y presentar un caso de supervivencia a

22. Alan Dent considera que el final de Waterloo Bridge es «as though Anna Karenina had fallen under the wheels of a Streetcar named Desire» (Dent, 1969, p. 123).

23. La filmografía de Vivien Leigh consta solamente de diecinueve películas, debido a sus numerosas actividades teatrales y a su mala salud -la actriz sufrió tuberculosis y bipolaridad-. 
un público hundido en una crisis económica desesperante. Lo más interesante en el caso de estos dos films es que esta dimensión propagandística es el resultado no solamente de un mero compromiso político o social, sino del trabajo de auténticos autores en simbiosis con su época. El hecho de dar una imagen positiva de una prostituta, es decir de un ser a menudo estigmatizado por la sociedad, para demostrar su pureza e incluso una cierta inocencia, es un rasgo típico del cine de James Whale que se confirma a partir de su película siguiente, Frankenstein (1931). Del mismo modo, la dimensión propagandística de la película de Mervyn LeRoy proviene de la propia puesta en escena de este director que inició, lo hemos dicho, el cine negro y que participa, con Waterloo Bridge, en un encuentro fructífero entre este género y el melodrama que va a determinar el cine de los años cuarenta. Proviene también de la encarnación de una actriz -en un momento clave de su evolución artística- autora de una filmografía en la que los temas de la decadencia física, moral, social y de una desesperación suicida, van a ser determinantes. Por todas estas razones, es necesario volver a estudiar estos Waterloo Bridge como dos hitos en la historia del cine hollywoodiense.

\section{Referencias bibliográficas}

Alion, Y. (03/2012). Avant-scène Cinéma 591, 132-133.

Bourget, J-L. (1994). Le mélodrame hollywoodien. Paris: Ramsay.

Curtis, J. (2003). James Whale, A New World of Gods and Monsters. Minnesota: University of Minnesota Press Edition.

Dent, A. (1969). Vivien Leigh: A Bouquet. London: Hamish Hamilton.

LeRoy, M. (1974). Take One. London, New York: W. H. Allen.

Mainer, C. (2013). El cine nortemaericano durante la Gran Depresión (19291939). Fotocinema 6, 171-200.

Masson, A. (05/2012). Positif 615, 78.

Mattéi, J-P. (12/1998). Paul Fejos, James Whale: des conquérants de Nouveau Monde. 1895, 26, 173-175.

McLean, A. (2008). Dying Swans and Madmen: Ballet, the Body and Narrative cinema. New Brunswick, New Jersey, London: Rutgers University Press.

Mouren, Y. (2005). Le flash-back. Paris: Armand Colin. 
Sherwood, R. E. (1930). Waterloo Bridge. New York, London: Charles Scribner's sons.

Silver, A \& Ward, E. (1987). Encyclopédie du film noir. Paris: Rivages

Walker, A. (1994). Vivien: The Life of Vivien Leigh. London: Orion.

\section{DVD}

LeRoy, M. (2012). La valse dans l'ombre. Paris: Wild Side Vidéo. 